\title{
Dynamic Modulation of Inspiratory Drive Currents by Protein Kinase A and Protein Phosphatases in Functionally Active Motoneurons
}

\author{
Christopher M. Bocchiaro, ${ }^{1}$ Shane A. Saywell, ${ }^{2}$ and Jack L. Feldman ${ }^{2}$ \\ Systems Neurobiology Laboratory, Departments of ${ }^{1}$ Physiological Science and ${ }^{2}$ Neurobiology, David Geffen School of Medicine, University of California Los \\ Angeles, Los Angeles, California 90095-1763
}

\begin{abstract}
Plasticity underlying adaptive, long-term changes in breathing behavior is hypothesized to be attributable to the modulation of respiratory motoneurons by intracellular second-messenger cascades. In quiescent preparations, protein kinases, including cAMP-dependent protein kinase A (PKA), potentiate glutamatergic inputs. However, the dynamic role of protein kinases or phosphatases in functionally active and behaviorally relevant preparations largely remains to be established. Rhythmic inspiratory drive to motoneurons innervating inspiratory muscles is mediated by the release of glutamate acting predominately on AMPA receptors. In rhythmically active brainstem slices from neonatal rats, we investigated whether synaptic AMPA receptor function could be modulated by changes in intracellular PKA activity, affecting inspiratory drive in hypoglossal (XII) motoneurons. Intracellular perfusion of the catalytic subunit of PKA potentiated endogenous synaptic and (exogenously applied) AMPA-induced currents in XII motoneurons. Conversely, when a peptide inhibitor of PKA was perfused intracellularly, inspiratory drive currents were depressed. Intracellular perfusion with microcystin, a potent phosphatase 1 and 2 a inhibitor, increased both endogenous and exogenous AMPA receptor-mediated currents, further supporting a role of phosphorylation in modulating motoneuronal excitability affecting behaviorally relevant synaptic inputs. These findings suggest that PKA is constitutively active in XII motoneurons in vitro. Thus, endogenous synaptic AMPA currents in XII motoneurons are influenced by phosphorylation, specifically by PKA, and dephosphorylation. The role of this modulation may be to keep the activity of motoneurons within a dynamic range that aids in responding to different physiological challenges affecting breathing, such as exercise, hypoxia, and sleep.
\end{abstract}

Key words: excitability; respiration; preBötzinger; plasticity; PKA; AMPA; phosphatase; sleep apnea

\section{Introduction}

Established models of neuronal plasticity, such as long-term potentiation (LTP) and long-term depression (LTD) seen in hippocampal, cerebellar, and cortical neurons (Colwell and Levine, 1995; Kameyama et al., 1998; Lee et al., 2000; Snyder et al., 2000), focus on the modulation of glutamatergic inputs in isolated neurons or in slices in which activity is induced pharmacologically or by electrical stimulation. These forms of synaptic plasticity appear to be mediated, at least in part, by protein kinases that phosphorylate AMPA receptor subunits to alter their conductance (Derkach et al., 1999) or affect the shuttling of AMPA receptors to and from the postsynaptic membrane (Beattie et al., 2002). One such protein kinase, PKA, phosphorylates AMPA/kainate receptors in both neuronal and cell culture systems (Greengard et al., 1991; Wang et al., 1991; Kameyama et al., 1998; Carvalho et al., 1999; Lee et al., 2000). Phosphorylation-induced increases in excitatory synaptic transmission affect neurons in the neostriatum

Received Sept. 19, 2002; revised Nov. 15, 2002; accepted Nov. 21, 2002.

This work was supported by National Institutes of Health Grant NS24742.

Correspondence should be addressed to Dr. Jack L. Feldman, Department of Neurobiology, David Geffen School of Medicine, University of California Los Angeles, Box 951763, Los Angeles, CA 90095-1763. E-mail: feldman@ucla.edu. Copyright $\odot 2003$ Society for Neuroscience $\quad 0270-6474 / 03 / 231099-05 \$ 15.00 / 0$ and hippocampus in vitro (Colwell and Levine, 1995; Lee et al., 2000), and in the brainstem and striatum in vivo (Lalley et al., 1997; Snyder et al., 2000). Also, inhibiting protein phosphatases 1 and 2 a potentiates peak glutamate and AMPA currents in neurons in both cortical (Thomas et al., 1997) and brainstem (Ge and Feldman, 1998) slices.

In quiescent preparations, protein kinases, including PKA, potentiate glutamatergic inputs; however, the dynamic role of protein kinases or phosphatases in functionally active and behaviorally relevant neurons largely remains to be established. Motoneurons receive excitatory amino acid input from premotor neurons and transform this activity into appropriate output to produce muscle contraction. Delineating how motoneuronal excitability is controlled is an important component in understanding behavior (Rekling et al., 2000).

The principal fast excitatory inputs to motoneurons, like most other neurons, are glutamatergic (Rekling et al., 2000), yet whether motoneurons can show synaptic plasticity similar to hippocampal, striatal, and cortical neurons is rarely considered. We investigated the role of PKA and protein phosphatases 1 and $2 \mathrm{a}$ in modulating the excitability of hypoglossal (XII) motoneurons, which innervate the tongue and participate in breathing (by modulating upper airway resistance) and oral-motor behaviors, 
including swallowing, phonation, and mastication. The inability to properly modulate XII motoneuronal excitability during sleep appears to be a major contributor to snoring and obstructive sleep apnea (OSA) (Fogel et al., 2000). Factors that exacerbate OSA, such as alcohol consumption (Scanlan et al., 2000), may also regulate synaptic plasticity through PKA-dependent mechanisms (Melis et al., 2002). In response to episodic hypoxia in vivo, activation of second-messenger cascades in motoneurons is hypothesized to underlie adaptive activity-independent respiratory plasticity (Baker-Herman and Mitchell, 2002).

XII motoneurons receive inspiratory-related excitatory drive currents that are principally mediated by AMPA receptors (Funk et al., 1993). We patch-clamped XII motoneurons that receive endogenous inspiratory drive in medullary slices of neonatal rat and examined the effects of manipulating intracellular PKA and phosphatase activity on endogenous synaptic inspiratory drive. We found that modulation of AMPA receptors by PKA and protein phosphatases dramatically affects the excitability of these functionally active motoneurons.

\section{Materials and Methods}

Experiments were performed on a medullary slice preparation that spontaneously generates respiratory rhythm (Smith et al., 1991). Neonatal Sprague Dawley rats ( $0-3 \mathrm{~d}$ of age) were anesthetized by hypothermia and rapidly decerebrated. The brainstem-spinal cord was isolated, mounted rostral side up in the specimen vise of a Vibratome, and sectioned serially in the transverse plane until the nucleus ambiguus and inferior olive were visible. One transverse slice (600-700 $\mu \mathrm{m}$ thick) containing the preBötzinger complex was cut. The slice was transferred to a recording chamber of $1 \mathrm{ml}$ volume. The dissection and slicing were performed in artificial CSF (ACSF) bubbled with $95 \% \mathrm{O}_{2}-5 \% \mathrm{CO}_{2}$ at room temperature. The ACSF contained (in $\mathrm{mM}$ ): $128 \mathrm{NaCl}, 3.0 \mathrm{KCl}, 1.5 \mathrm{CaCl}_{2}$, $1 \mathrm{MgSO}_{4}, 23.5 \mathrm{NaHCO}_{3}, 0.5 \mathrm{NaH}_{2} \mathrm{PO}_{4}$, and 30 glucose. During electrophysiological recording, the slice was continuously superfused $(3 \mathrm{ml} /$ min) with ACSF (temperature maintained at $27 \pm 1^{\circ} \mathrm{C}$ ) with elevated $\mathrm{KCl}(9 \mathrm{~mm})$ to assure robust rhythmic respiratory activity.

Electrophysiological recording. XII motoneurons were visualized with infrared differential interference contrast microscopy. XII motoneurons were identified by the criteria of Funk et al. (1993). All XII motoneurons selected for this study received endogenous rhythmic activity in phase with XII nerve (XIIn) inspiratory-modulated motor output. Electrodes (4-8 $\mathrm{M} \Omega ; \sim 1.5 \mu \mathrm{m}$ tip diameter) were pulled from borosilicate glass on a horizontal puller. Electrodes were filled with solution containing (in mM): 120 potassium gluconate, 11 EGTA, $5 \mathrm{NaCl}, 1 \mathrm{CaCl}_{2}, 10 \mathrm{HEPES}$, and $2 \mathrm{ATP}\left(\mathrm{Mg}^{2+}\right.$ salt), $\mathrm{pH}$ adjusted to 7.3 with $\mathrm{KOH}$. Positive pressure was applied to the back of the electrode as it was advanced into the tissue. When the electrode was positioned on the surface of the soma of the target neuron, positive pressure was released and negative pressure was applied to form a Giga $\Omega$ seal. The cell was then ruptured with short negative pressure pulses. Intracellular signals were amplified with a patch-clamp amplifier (Axopatch 1D, Axon Instruments, Foster City, CA); whole-cell capacitance was compensated, as was the series resistance. Input resistance stability was checked throughout experiments. During voltageclamp recordings, XII motoneurons were held at $-70 \mathrm{mV}$.

Respiratory-related nerve activity was recorded from the cut ends of the XII nerve (XIIn) with a suction electrode, amplified 10,000 or 20,000 times, bandpass filtered $(3-3000 \mathrm{~Hz})$, and rectified. XIIn burst activity defined the inspiratory period.

Drugs and drug application. The following drugs (obtained from Sigma, St. Louis, MO) were prepared in patch electrode solution: active catalytic subunit of PKA (250 U/ml; P2645; Sigma); inhibitory peptide (PKI) (500 $\mu \mathrm{g} / \mathrm{ml}$; P0300; Sigma), which binds to the catalytic subunit of PKA and inhibits its activity (Raymond et al., 1993); and microcystin-LR (MLR; $50 \mu \mathrm{M}$ ), dissolved in dimethylsulfoxide (DMSO) and diluted before use (final DMSO, $0.5 \% \mathrm{v} / \mathrm{v}$ ).

For experiments involving application of exogenous AMPA, action potential-dependent synaptic transmission was prevented by bath application of tetrodotoxin (TTX, $1 \mu \mathrm{M}$ ). AMPA was dissolved in ACSF (10 $\mu \mathrm{M})$ and pressure ejected from glass electrodes (tip diameter, $3 \mu \mathrm{m}$; pressure, 10 psi; duration, $50-150 \mathrm{msec}$ ) placed under visual guidance above the neuron being recorded. With this concentration and injection protocol (see below), we did not see any evidence of desensitization of AMPA responses between injections.

Data acquisition and analysis. Signals were recorded digitally (DC, 20 $\mathrm{kHz}$ ) and stored on a computer hard drive for off-line analysis using Clampfit 8.0 (Axon Instruments) and Origin 6.0 (Origin Lab Corp.). Statistical values are reported as means \pm SD. Differences between means were calculated by independent $t$ test, and $p<0.05$ was considered significant.

Recordings of endogenous synaptic drive currents were triggered off the onset of the integrated XII motor output. Peak inspiratory drive currents were averaged from 6 to 10 consecutive inspiratory bursts. Averaged peak currents were normalized to current amplitude immediately after whole-cell break-in. After establishing whole-cell patch configuration, membrane currents remained stable for the duration of the experiments, with no significant rundown or change in input resistance.

\section{Results}

\section{Effects on endogenous inspiratory drive}

When whole-cell voltage-clamped with microelectrodes filled with standard patch solution, XII motoneurons exhibit periodic inward currents in-phase with integrated XII motor output (Funk et al., 1993). We observed no change in the shape and no decrement in peak amplitude of these currents in recordings lasting up to $30 \mathrm{~min}$ (Fig. $1 \mathrm{~A}$, top inset, Control).

To establish a role for PKA in modulating endogenous respiratory drive, we included a purified peptide of the PKA catalytic subunit in the patch electrode. This resulted in progressive increases in peak inspiratory drive currents in XII motoneurons after break-in (Fig. $1 A$ ). Normalized (to break-in values) peak currents increased to $190 \pm 60 \%$ (Fig. 1 A, bottom inset) $(p<$ $0.01)$, reaching peak amplitude in $\sim 15 \min (n=7)$.

To determine whether endogenous PKA activity affected inspiratory drive currents under our experimental conditions, we recorded with PKI in the patch electrode. Peak endogenous currents decreased $56 \pm 13 \%$ (Fig. 1 B, bottom inset) $(p<0.01)$ relative to break-in values in a time-dependent manner starting at $4 \mathrm{~min}$ after and peaking $10 \mathrm{~min}$ after break-in (Fig. $1 B)(n=5)$.

Because changes in intracellular PKA activity affected inspiratory drive currents, we tested whether changes in phosphatase activity had consistent effects. Inclusion of microcystin, a membrane-impermeable inhibitor of protein phosphatases 1 and $2 \mathrm{a}$, in the patch electrode increased peak endogenous inspiratory drive currents in XII motoneurons (Fig. 1C). Peak currents increased to $170 \pm 21 \%$ (Fig. $1 C$, bottom inset) $(p<0.001)$, reaching peak amplitude $5-30$ min after break-in $(n=5)$.

\section{Effects on exogenous AMPA application}

To demonstrate the effect of phosphorylation on AMPA receptor-mediated currents, XII motoneurons were identified as indicated above, and then AMPA $(10 \mu \mathrm{M})$ was applied exogenously (via micropipette) after bath application of TTX $(1 \mu \mathrm{M})$. As TTX washed into the recording chamber, rhythmic inspiratory XII nerve activity ceased, as did rhythmic inspiratory drive currents in XII motoneurons. The duration of AMPA injections was adjusted to produce peak inward currents with a shape and peak magnitude similar to endogenous inspiratory currents. Exogenously applied AMPA-induced currents were longer lasting than endogenous inspiratory drive currents, presumably because of the effects of diffusion of pressure-ejected AMPA through the brainstem tissue (Funk et al., 1995). Injections were spaced at 

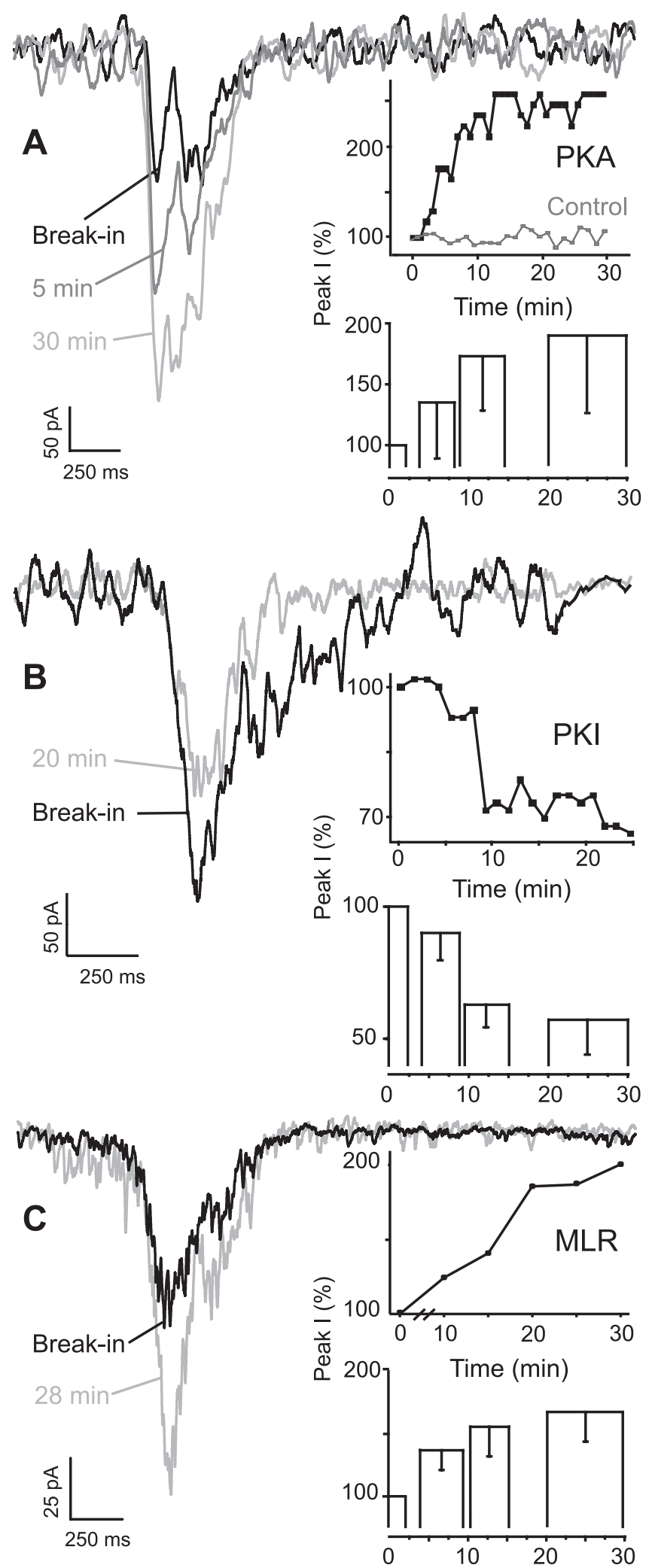

Figure 1. Effects of PKA and phosphatase manipulation on inspiratory drive currents. $A$, Effect of intracellular dialysis of activated PKA enhanced peak endogenous glutamatergic inspiratory drive currents in XII motoneurons. Potentiation of synaptic drive currents of a PKA dialyzed XII motoneuron 1, 5, and 30 min after establishing whole-cell recording (break-in; $V_{\mathrm{h}}=-70 \mathrm{mV}$ ) is shown. Traces are averaged $(n=10)$. Inset, top, Time course (for traces shown at left) of peak whole-cell inspiratory drive current in an XII motoneuron in response to dialysis with PKA $(250 \mathrm{U} / \mathrm{ml})$. Control time course without PKA demonstrates the stability of recordings. Bottom, Group data $(n=7)$ histogram for XII motoneurons dialyzed with activated PKA. Here and elsewhere, histogram widths reflect the temporal epochs that were averaged. $B$, least 1 min apart to avoid AMPA receptor desensitization (Trussell et al., 1988).

Inclusion of the PKA catalytic subunit in the patch electrode enhanced the peak AMPA-induced current $165 \pm 36 \%$ (Fig. 2 A, bottom inset) $(n=3 ; p<0.05)$. Potentiation of AMPA currents developed slowly over several minutes to a peak value, reaching a maximum 5-16 min after break-in (Fig. 2A).

To determine whether phosphatase inhibition had the same effect on AMPA currents as on endogenous inspiratory drive currents, we performed the above protocol with microcystin (50 $\mu \mathrm{M})$ in the patch electrode. We observed significantly increased peak whole-cell currents in response to local exogenous AMPA application (Fig. 2 B, bottom inset) $(202 \pm 45 \%$; $n=5 ; p<0.001)$. Maximal effects of microcystin occurred within $15 \mathrm{~min}$ of break-in.

\section{Discussion}

Our main finding is that a change in PKA activity in XII motoneurons modulates their AMPA receptor-mediated synaptic currents. In XII motoneurons, enhancement of PKA activity (by either increased intracellular PKA or decreased phosphatase activity) increased endogenous synaptic inspiratory drive currents as well as those induced by exogenously applied AMPA; similarly, inhibiting PKA activity depressed these currents.

Changes in the phosphorylation state of ionotropic glutamate receptor subunits (GluRs) contribute to some forms of neuronal plasticity, such as LTP and LTD (Roche et al., 1994; Roberson and Sweatt, 1996; Carvalho et al., 2000; Soderling and Derkach, 2000). PKA phosphorylates both native and recombinant AMPA/kainate receptors (Greengard et al., 1991; Wang et al., 1991; Kameyama et al., 1998; Carvalho et al., 1999); phosphorylation of GluR1 subunits by PKA potentiates glutamate-activated currents (Roche et al., 1996). Potentiation of glutamate-mediated synaptic currents resulting from PKA activation is seen in neurons in slices of hippocampus (Lee et al., 2000) and of striatum (Colwell and Levine, 1995) and in in vivo brainstem (Lalley et al., 1997). Inhibition of phosphatases 1 and 2a increases the amplitude of glutamatergic currents (Figurov et al., 1993; Thomas et al., 1997; Ge and Feldman, 1998), suggesting that basal phosphatase activity modulates receptor activity under these various experimental conditions.

Phosphorylation of AMPA receptors in inspiratory, including XII, motoneurons is postulated to underlie compensatory changes in their excitability in response to physiological challenges. XII motoneurons contribute to the regulation of upper airway resistance, and their failure to produce appropriate output during sleep results in airway collapse. This can cause snoring and OSA (Fogel et al., 2000), which is characterized by intermittent

$\leftarrow$

Intracellular dialysis of PKI diminished peak inspiratory drive currents in XII motoneurons. Decreased endogenous currents of XII motoneuron dialyzed with PKI immediately after establishing recording and $20 \mathrm{~min}$ later are shown $(n=10)$. Inset, top, Time course of normalized peak currents from an XII motoneuron dialyzed with PKI $(500 \mu \mathrm{g} / \mathrm{ml})$. Bottom, Group data $(n=5)$ histogram for XII motoneurons dialyzed with activated PKI. C, Intracellular dialysis of MLR enhanced endogenous inspiratory drive in XII motoneurons. An average of six consecutive traces for an XII motoneuron dialyzed with microcystin $(50 \mu \mathrm{m})$ immediately after whole-cell break-in and at $28 \mathrm{~min}$ after establishing recording is shown. Inset, top, Time course of peak whole-cell current in an XII motoneuron in response to dialysis with microcystin. Bottom, Group data ( $n=$ 5) histogram for XII motoneurons dialyzed with MLR. Peak inspiratory current values are normalized to amplitude immediately after break-in [Peak I(\%)]. Top inset graphs correspond to the traces shown at left for each panel. Histograms represent pooled, averaged, and normalized data comparing peak currents obtained immediately after break-in with maximal enhancement of current magnitude at times indicated; error bars indicate so. 

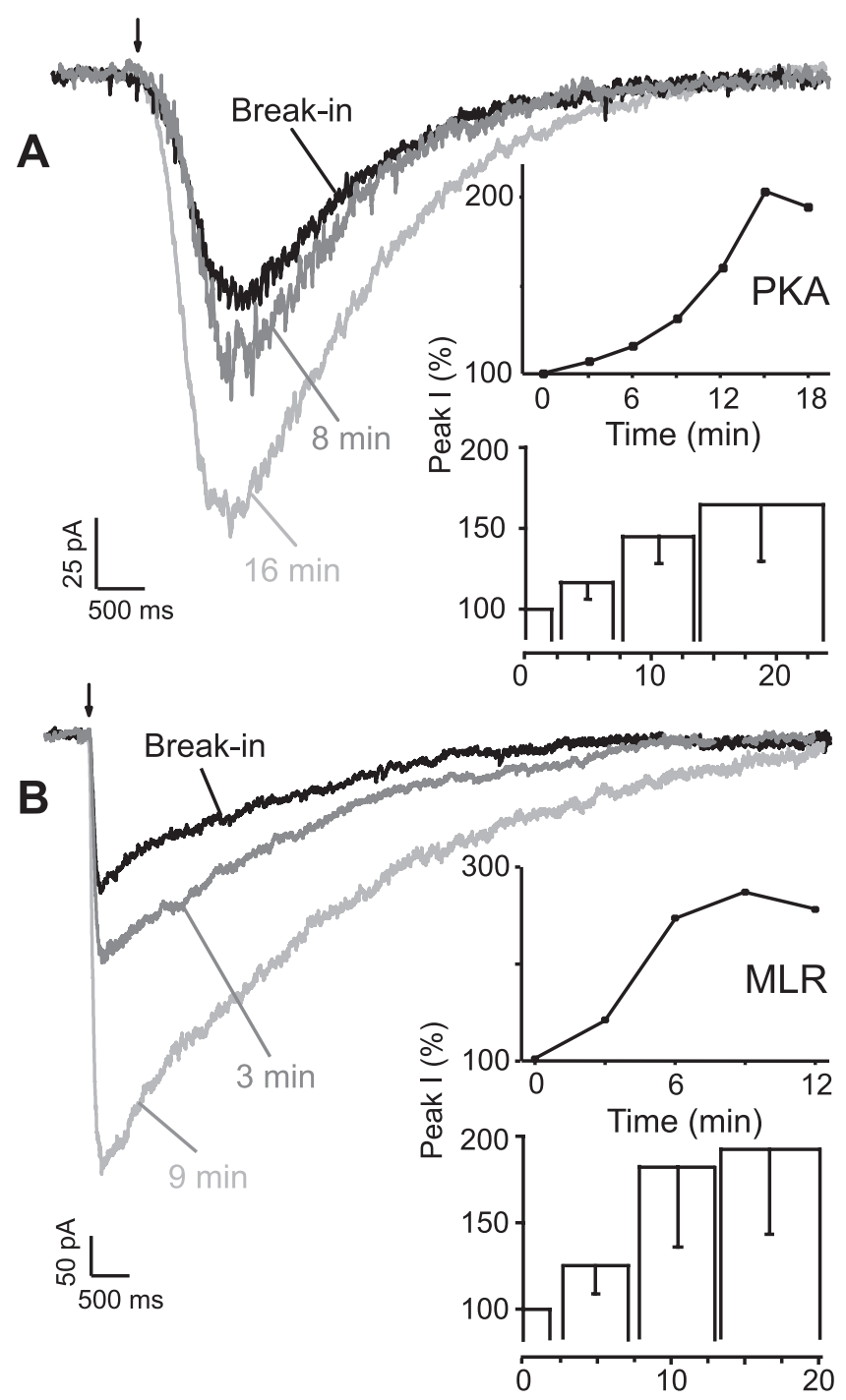

Figure 2. Effects of phosphorylation on exogenous AMPA currents. A, Intracellular dialysis of PKA potentiated peak AMPA currents in XII motoneurons. Whole-cell currents produced by focal exogenous AMPA (arrow, $10 \mu \mathrm{M}, 100 \mathrm{msec}$ ) in the presence of TTX (1 $\mu \mathrm{M}$ ) immediately after break-in followed by 8 and $16 \mathrm{~min}$ in an XII motoneuron $\left(V_{\mathrm{h}}=-70 \mathrm{mV}\right)$ are shown. Inset, top, Normalized peak AMPA currents (for traces shown at left) after break-in and dialysis of PKA into XII motoneurons. Bottom, Group data $(n=3)$ histogram for AMPA currents elicited in XII motoneurons dialyzed with activated PKA. B, Potentiated peak AMPA currents in XII motoneurons dialyzed with MLR. AMPA currents triggered by AMPA application (arrow) in the presence of TTX at times indicated during dialysis with MLR are shown. Inset, top, Time course of peak AMPA current in an XII motoneuron in response to MLR. Bottom, Group data $(n=5)$ histogram of normalized peak AMPA currents in MLR dialyzed XII motoneurons. Top inset graphs correspond to the traces shown at left for each panel. Histograms represent pooled, averaged, and normalized data comparing peak currents obtained immediately after break-in with maximal enhancement of current magnitude at times indicated; error bars indicate so.

episodes of upper airway obstruction, leading to repetitive bouts of hypoxia. When exposed to repetitive episodes of hypoxia, there are marked and long-lasting increases in XII motoneuronal activity in vivo (Bach and Mitchell, 1996). Phosphorylation of glutamate receptors, leading to potentiation of ionic currents, is hypothesized to underlie these changes in XII motoneuronal activity (Fuller et al., 2000).

We have shown that AMPA currents from functionally active synapses in XII motoneurons are regulated by PKA and protein phosphatases, and that PKA is constitutively active in XII motoneurons in vitro. In in vivo experiments investigating the role of
PKA in the neural control of breathing (Lalley et al., 1997; Richter et al., 1997), the effects of membrane permeant drugs on respiratory neurons of indeterminate function in the ventral respiratory group were studied; the site of action of these drugs, especially presynaptic versus postsynaptic, could not be ascertained. Here, we dialyzed membrane impermeant drugs into respiratory neurons of known function (i.e., XII motoneurons) and then studied these neurons after synaptic isolation with TTX. Thus, we could observe postsynaptic responses clearly affecting AMPA receptors. In addition, in vitro we could obtain voltage-clamp recordings that were not contaminated by fast $\mathrm{Na}^{+}$currents, as was the case in vivo (Lalley et al., 1997) and is suggestive of poor space clamp.

The intracellular balance between protein kinases and phosphatases determines the basal level of phosphorylation (Carvalho et al., 1999). The general role of this modulation in XII motoneurons may be to adjust their excitability (Carvalho et al., 1999) to assure appropriate contraction and relaxation of tongue muscles to different physiological challenges affecting breathing, such as exercise, hypoxia (at altitude or during lung disease), and sleep, and its dysfunction could underlie the failure to produce appropriate output, such as occurs in many cases of snoring and OSA. The endogenous signals that contribute to the upregulation or downregulation of PKA and associated phosphatases have not yet been determined. Similar modulation of the excitability of other types of motoneurons would represent an important component of behavioral adaptation of movements to challenges affecting motor performance (Rekling et al., 2000).

\section{References}

Bach KB, Mitchell GS (1996) Hypoxia-induced long-term facilitation of respiratory activity is serotonin dependent. Respir Physiol 104:251-260.

Baker-Herman TL, Mitchell GS (2002) Phrenic long-term facilitation requires spinal serotonin receptor activation and protein synthesis. J Neurosci 22:6239-6246.

Beattie EC, Stellwagen D, Morishita W, Bresnahan JC, Ha BK, Von Zastrow M, Beattie MS, Malenka RC (2002) Control of synaptic strength by glial TNF $\alpha$. Science 295:2282-2285.

Carvalho AL, Kameyama K, Huganir RL (1999) Characterization of phosphorylation sites on the glutamate receptor 4 subunit of the AMPA receptors. J Neurosci 19:4748-4754.

Carvalho AL, Duarte CB, Carvalho AP (2000) Regulation of AMPA receptors by phosphorylation. Neurochem Res 25:1245-1255.

Colwell CS, Levine MS (1995) Excitatory synaptic transmission in neostriatal neurons: regulation by cAMP-dependent mechanisms. J Neurosci 15:1704-1713.

Derkach V, Barria A, Soderling TR (1999) $\mathrm{Ca}^{2+} /$ calmodulin-kinase II enhances channel conductance of $\alpha$-amino-3-hydroxy-5-methyl-4isoxazolepropionate type glutamate receptors. Proc Natl Acad Sci USA 96:3269-3274.

Figurov A, Boddeke H, Muller D (1993) Enhancement of AMPA-mediated synaptic transmission by the protein phosphatase inhibitor calyculin A in rat hippocampal slices. Eur J Neurosci 5:1035-1041.

Fogel RB, Malhotra A, Shea SA, Edwards JK, White DP (2000) Reduced genioglossal activity with upper airway anesthesia in awake patients with OSA. J Appl Physiol 88:1346-1354.

Fuller DD, Bach KB, Baker TL, Kinkead R, Mitchell GS (2000) Long term facilitation of phrenic motor output. Respir Physiol 121:135-146.

Funk GD, Smith JC, Feldman JL (1993) Generation and transmission of respiratory oscillations in medullary slices: role of excitatory amino acids. J Neurophysiol 70:1497-1515.

Funk GD, Smith JC, Feldman JL (1995) Modulation of neural network activity in vitro by cyclothiazide, a drug that blocks desensitization of AMPA receptors. J Neurosci 15:4046-4056.

Ge Q, Feldman JL (1998) AMPA receptor activation and phosphatase inhibition affect neonatal rat respiratory rhythm generation. J Physiol (Lond) 509:255-266.

Greengard P, Jen J, Nairn AC, Stevens CF (1991) Enhancement of the glu- 
tamate response by cAMP-dependent protein kinase in hippocampal neurons. Science 253:1135-1138.

Kameyama K, Lee HK, Bear MF, Huganir RL (1998) Involvement of a postsynaptic protein kinase A substrate in the expression of homosynaptic long-term depression. Neuron 21:1163-1175.

Lalley PM, Pierrefiche O, Bischoff AM, Richter DW (1997) cAMPdependent protein kinase modulates expiratory neurons in vivo. J Neurophysiol 77:1119-1131.

Lee HK, Barbarosie M, Kameyama K, Bear MF, Huganir RL (2000) Regulation of distinct AMPA receptor phosphorylation sites during bidirectional synaptic plasticity. Nature 405:955-959.

Melis M, Camarini R, Ungless MA, Bonci A (2002) Long-lasting potentiation of GABAergic synapses in dopamine neurons after a single in vivo ethanol exposure. J Neurosci 22:2074-2082.

Raymond LA, Blackstone CD, Huganir RL (1993) Phosphorylation and modulation of recombinant GluR6 glutamate receptors by cAMPdependent protein kinase. Nature 361:637-641.

Rekling JC, Funk GD, Bayliss DA, Dong XW, Feldman JL (2000) Synaptic control of motoneuronal excitability. Physiol Rev 80:767-852.

Richter DW, Lalley PM, Pierrefiche O, Haji A, Bischoff AM, Wilken B, Hanefeld F (1997) Intracellular signal pathways controlling respiratory neurons. Respir Physiol 110:113-123.

Roberson ED, Sweatt JD (1996) Transient activation of cyclic AMPdependent protein kinase during hippocampal long-term potentiation. J Biol Chem 271:30436-30441.
Roche KW, Tingley WG, Huganir RL (1994) Glutamate receptor phosphorylation and synaptic plasticity. Curr Opin Neurobiol 4:383-388.

Roche KW, O’Brien RJ, Mammen AL, Bernhardt J, Huganir RL (1996) Characterization of multiple phosphorylation sites on the AMPA receptor GluR1 subunit. Neuron 16:1179-1188.

Scanlan MF, Roebuck T, Little PJ, Redman JR, Naughton MT (2000) Effect of moderate alcohol upon obstructive sleep apnoea. Eur Respir J 16:909-913.

Smith JC, Ellenberger HH, Ballanyi K, Richter DW, Feldman JL (1991) PreBotzinger complex: a brainstem region that may generate respiratory rhythm in mammals. Science 254:726-729.

Snyder GL, Allen PB, Fienberg AA, Valle CG, Huganir RL, Nairn AC, Greengard P (2000) Regulation of phosphorylation of the GluR1 AMPA receptor in the neostriatum by dopamine and psychostimulants in vivo. J Neurosci 20:4480-4488.

Soderling TR, Derkach VA (2000) Postsynaptic protein phosphorylation and LTP. Trends Neurosci 23:75-80.

Thomas GD, O’Rourke B, Sikkink R, Rusnak F, Marban E, Victor RG (1997) Differential modulation of cortical synaptic activity by calcineurin (phosphatase 2B) versus phosphatases 1 and 2A. Brain Res 749:101-108.

Trussell LO, Thio LL, Zorumski CF, Fischbach GD (1988) Rapid desensitization of glutamate receptors in vertebrate central neurons. Proc Natl Acad Sci USA 85:4562-4566.

Wang LY, Salter MW, MacDonald JF (1991) Regulation of kainate receptors by cAMP-dependent protein kinase and phosphatases. Science 253:11321135. 